\title{
ARGUMENTO A CONTRARIO Y REGLAS CONSTITUTIVAS
}

\section{Introducción}

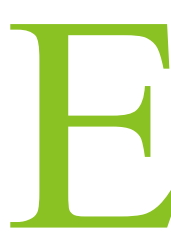

n este trabajo quiero exponer algunas consideraciones en relación con la controvertida cuestión del uso de instrumentos lógicos en el derecho y, en especial, en el razonamiento de los jueces expresado en forma silogística (el llamado silogismo dicástico). Los términos generales del debate son bien conocidos y no es cuestión de proponerlos aquí de nuevo, ni tampoco de tratar de establecer un balance de los resultados ${ }^{1}$. Mis consideraciones, dicho muy brevemente, se insertan en la temática que llama la atención de los juristas acerca de la pregunta de si la (y cuál) lógica es utilizada por el juez para decidir las controversias que se le someten en cuanto órgano autorizado por el ordenamiento jurídico.

Naturalmente, habría que esclarecer previamente la cuestión de si, dada la particular naturaleza del lenguaje utilizado en la formulación de las premisas del razonamiento judicial (o, mejor, de una de ellas: la mayor), se necesita una lógica particular, una lógica del lenguaje normativo. Pues bien: me parece que, en virtud de la finalidad de este escrito, podemos omitir entrar en los aspectos particulares del problema del lenguaje normativo del razonamiento de los jueces, ya que -como se sabe- la literatura sobre este asunto es ampliamente conocida y, en consecuencia, se correría el riesgo de recorrer caminos ya muy trillados. No me interesaré pues por este ámbito; mis observaciones deben entenderse, por tanto, en sus nexos con la lógica clásica a dos valores.

Dando pues como resuelto el problema relativo a la presencia de entidades no apofánticas en el silogismo judicial ${ }^{2}$, la cuestión que sobre todo me interesa es otra, la cual -en mi opiniónha suscitado relativamente poca

\footnotetext{
${ }^{1}$ Balances, en este sentido, existen varios en la literatura; sólo como ejemplo, cito Roberto J. Vernengo, «Derecho y lógica: un balance provisorio», Anuario de Filosofia del Derecho, Instituto Nacional de Estudios Jurídicos, Madrid, 1987, pp. 303-329.

${ }^{2}$ Soy consciente, no obstante, de que tal vez éste sea el problema central de la cuestión de la aplicabilidad de la lógica al razonamiento de los jueces.
} 
atención en la literatura iusfilosófica reciente ${ }^{3}$. Me refiero, dicho muy simplemente, a la dificultad que surge si se debe demostrar, mediante el análisis lógico, que una conclusión judicial de signo negativo (la absolución de un imputado, por ejemplo) se sigue deductivamente de las premisas. El problema, como se sabe, es que se debe constatar que la inferencia a contrario constituye el clásico non sequitur, y que la conclusión de una inferencia de esta naturaleza no se puede defender con razones lógicas, mientras que -a pesar de todo- la conclusión parece ser razonablemente aceptable ${ }^{4}$. Supongamos, por ejemplo, que Fulano ha sido sometido a un proceso como acusado de haber cometido homicidio en la persona de Zutano. Si mediante el proceso se prueba que él efectivamente ha cometido ese delito, con la regla de inferencia modus ponens podemos establecer que la sanción prevista se sigue lógicamente de las premisas; pero el problema es: ¿qué sucede si, por el contrario, el proceso concluye que Fulano no ha cometido el delito? ¿De qué manera podemos establecer desde el punto de vista lógico (si es que podemos) que a él no se le debe imputar la sanción prevista por la disposición normativa que regula el caso? Como sabemos, nosotros podemos, razonablemente, establecer que si Fulano no ha cometido el hecho delictivo del que se le acusa no hay razón para que él deba sufrir la pena prevista por la ley; $y$, sin embargo, también sabemos que desde el punto de vista lógico caeríamos en la falacia conocida como la negación del antecedente si tratásemos de explicar la cuestión mediante un esquema lógico del tipo: «Todos aquellos que cometan homicidio deben ser condenados», $\mathrm{y}$ «Fulano no ha cometido homicidio», luego «Fulano no debe ser condenado». Entre las excepciones: M. Henket, «On the Logical Analysis of Judicial Decisions», en International Journal for the Semiotics of Law, 1992, V/14, pp. 153-164; J.H. Nieuwenhuis,

\footnotetext{
3 «Legitimatie en Heuristiek van het rechterlijt oordel», en Rechtsgeleerd Magazijn Themis, 1976, pp. 494-515; H. Kaptein, «Logica in reschtspraak, in het bijzonder in unalogie- en a contrarioredeneringen: vroom bedrog?», en F. H. van Eemeren y E. T. Feteris (eds.), Juridische Argumentatie in analyse, Gröningen, Wolters Noorhoof, 1991, pp. 75-90; I. Tammelo, «La logica del "petere" e del "quaerere" nel pensiero giuridico», en I. Tammelo e I. Tebaldeschi, Studi di logica giuridica, Milano, Giuffrè, 1976, pp. 79-86. Algunas huellas se pueden encontrar también en A. Costanzo, «Difficoltà della "reductio ad absurdum" e apparenti deroghe alla logica classica nelle argomentazioni giudiziali», en Rivista Internazionale di Filosofia del Diritto, 1990, 4, pp. 576-617, app. 584-86.

${ }^{4}$ La literatura sobre el Argumentum e contrario es, obviamente, muy vasta; sólo como ejemplos cito: A.G. Conte, «Completezza e chiusura», en Scritti in memoria de Widar Cesarini Sforza, Milano, Giuffrè, 1968, pp. 157-79, ahora en Id., Filosofia del linguaggio normativo I Studi 1965-1981, Torino, Giappichelli, 1989, pp. 31-53, espec, pp. 4352; E. García Máynez, Lógica del raciocinio jurídico, México, Distribuciones Fontamara, S. A., 1994, reimp. pp. 169 ss.; Ch. Perelman, Logique Juridique. Nouvelle Rhétorique, Paris, Dalloz, 1976, pp. 8 ss. y 55 ss., trad. it. Logica giuridica. Nuova retorica, Milano, Giuffrè, 1979; Id., con L. Olbrechts-Tyteca, Traité de l'argumentation. La nouvelle rhétorique, Paris, PUF, 1958, pp. 325, trad. it. Trattato dell'argomentazione. La nuova retorica, Torino, Einaudi, 1966, Véase también la ponencia de G. Tarello en el Congreso de Bruselas de 1971: «Die Juristische Argumentation», en Archiv für Rechts- un Sozialphilosophie, Neue Folge, no. 7, 1972, pp. 103-124.
} 
Pienso que no se puede dudar, antes que nada, de que el análisis lógico del razonamiento del juez ha sido tradicionalmente efectuado desde una perspectiva que parece privilegiar el fallo del juez que se manifiesta -por ejemplo, en el derecho penal- con una condena (o, en otras palabras, privilegiando el esquema deductivo que se configura cuando se utiliza la regla inferencial modus ponens), como en el clásico ejemplo de Kelsen: (A) «Todos los ladrones deben sufrir una condena», «Shulze es un ladrón», luego «Shulze debe sufrir una condena». Pero, ¿qué sucede si, por el contrario (como algunas veces acontece en la realidad judicial), nos encontrarnos con una conclusión del tipo «Shulze no debe sufrir una condena»?

Lo que intento -en pocas palabras- es proporcionar una razón ulterior para que el modelo formal de análisis del razonamiento judicial sea examinado con la máxima cautela, dado que -como resultará evidente- ese modelo oscurece aspectos relevantes del modo mediante el cual los jueces toman sus decisiones. Naturalmente, sosteniendo que el modelo formal deja fuera del análisis aspectos relevantes como la formación de las premisas, no digo nada fundamentalmente nuevo; me parece, en efecto, que ésta es una convicción bastante difundida entre los estudiosos del problema de la justificación de las decisiones judiciales. Sin embargo, mi investigación trata de examinar un aspecto del problema que tal vez puede arrojar mayor luz acerca de los límites de la aplicación de la lógica al razonamiento del juez.

Por «modelo formal» de razonamiento judicialentiendo aquella estructura de explicación del proceso de la toma de una decisión judicial que se interesa principalmente por la deducibilidad de la conclusión. No tengo la pretensión, obviamente, de proponer en estas pocas líneas una solución alternativa al modelo formal de razonamiento judicial ${ }^{5}$; me limito, más bien, a efectuar algunas observaciones mediante las cuales deseo contribuir a subrayar las dificultades de tal modelo de análisis.

\section{Decisión judicial: ¿Razonabilidad o análisis lógico?}

El problema consiste -repito- en el hecho de que, por un lado, mediante modus ponens establecernos la correcta forma lógica del silogismo dicástico

\footnotetext{
${ }^{5}$ Ya que, como sabemos, a esta tarea se han dedicado muchos autores; entre ellos: T. Viehweg, Topik und Jurisprudenz (1953), trad. it. a cargo de G. Crifò, Topica e giurisprudenza, Milano, Giuffrè, 1962; Ch. Perelman, Logique Juridique. Nouvelle Rhétorique, cit.; Ch. Perelman-L. Olbrechts -Tyteca, Traité de l'argumentation. La nouvelle rhétorique, cit.; A. Giuliani, Il concetto di prova. Contributo alla logica giuridica, Milano, Giuffrè, 1961; Id., «Logica (teoria dell'argomentazione)», voz de la Enciclopedia del diritto, XXV, Milano, Giuffrè, $1975 ; \mathrm{F}$. Cavalla, «Topica giuridica», voz de la Enciclopedia del diritto, XLIV, Milano, Giuffrè, 1992; L. Gianformaggio, Modelli di ragionamento giuridico. Modello deduttivo, modello induttivo, modello retorico (1983), ahora en Id., Studi sulla giustificazione giuridica, Torino, Giappichelli, 1986.
} 
con la conclusión de que Shulze debe sufrir una pena, mientras que, por el otro, la conclusión posible de que Shulze no deba sufrir tal pena parece configurar de alguna manera una paradoja, una aporía. Aporía que, entre otras cosas -como sostienen Tammelo y Tebaldeschi ${ }^{6}$ (entre los pocos que se han interesado por esta cuestión)- es sólo una entre las muchas que parecen surgir de la aplicación de las reglas de inferencia lógica. Otra aporía, en efecto, se derivaría del teorema lógico del ex falso quodlibet, el cual establece que de premisas contradictorias es posible deducir cualquier conclusión. Dado que no podemos excluir la presencia, en un ordenamiento jurídico, de dos disposiciones antinómicas válidas, una que prohíba y otra que prescriba el mismo comportamiento, se sigue que de la conjunción de éstas podríamos -lógicamente- inferir cualquier conclusión ${ }^{7}$.

Pero, para regresar a la cuestión específica que aquí se trata, el problema práctico es que -pienso que podernos estar de acuerdo- ningún tribunal superior encontrará irracional la decisión judicial que se funda sobre la negación del antecedente (razonamiento, como hemos visto, inválido desde el punto de vista lógico). Se hace necesario, entonces, analizar el problema, con el fin de verificar si es posible (y con qué instrumentos) encontrar una explicación lógica, o por el contrario -como me inclino a pensar- el instrumental lógico se demuestra insuficiente.

Deseo pues analizar brevemente algunas propuestas relativas a este problema, tratando de argumentar -como por lo demás parecen hacer quienes se han interesado por la cuestión ${ }^{8}$ - que a fin de cuentas el camino que hay que recorrer no es el de la lógica; al final avanzaré -con objetivos más bien exploratorios que resolutivos- mi propuesta.

\section{Negación del antecedente. Modus ponens}

Veamos pues algunas posibles soluciones. Es claro, de entrada, que para que en el esquema del razonamiento silogístico se dé una conclusión negativa una de sus premisas debe ser negativa; ¿pero cuál? Me parece que cualquier propuesta de solución en este sentido tiene que basarse en la negación del antecedente; naturalmente, ello se debe, además del requisito de carácter lógico, al hecho de que -en el caso del ejemplo- Shulze no es el autor del hecho concreto al que está ligada la sanción.

${ }^{6}$ Cfr. I. Tammelo, La logica del «petere» e del «quaerere» nel pensiero giuridico, cit., p. 85; I. Tebaldeschi, «Il metodo della isoformula nel ragionaniento giuridico», en I. Tammelo -I. Tebaldeschi, Studi di logica giuridica, cit., pp. 137-150, en pp. 139-140.

${ }^{7}$ Otras aporías se podrían derivar del uso del teorema lógico de la adición. Para el análisis de ésta, cfr. I. Tammelo, La logica del «petere» e del «quaerere» nel pensiero giuridico, cit., p. 85.

\footnotetext{
${ }^{8}$ Por ejemplo, Henket, On the Logical Analysis of Judicial Decisions, cit., pp. 160-164.
} 
La alternativa más plausible, entonces, es la de modificar la premisa menor, visto que si Shulze no debe sufrir la pena es porque -razonando con simple sentido común- él no es un ladrón 9 . Un posible esquema que resultaría sería el siguiente: (B) «Todos los ladrones deben sufrir una condena», «Shulze no es un ladrón», entonces «Shulze no debe sufrir una condena» ${ }^{10}$. Ahora bien, aunque aparentemente estamos en presencia de un silogismo válido, en realidad la conclusión no se sigue en absoluto de las premisas; en efecto, en la conclusión existe algo que, por el contrario, no existe en las premisas. Si representamos «ladrones» y «condena» respectivamente con L y $\mathrm{P}$, y Shulze con $\mathrm{x}$, tendremos que en la premisa mayor se afirma que la entera clase de los L debe incluirse en la de los $\mathrm{P}$, mientras que en la premisa menor se dice simplemente que $\mathrm{x}$ no pertenece a la clase L. Pero no tenemos proposición alguna (ni negativa ni positiva) relativa a la clase $\mathrm{P}$. Podemos entonces pensar que la clase $\mathrm{P}$ no se agote completamente en la clase L (o, en otras palabras, que la clase de quienes deben sufrir una condena comprenda otras clases, distintas de la clase de los ladrones). Luego, la conclusión del esquema B no es válida en virtud de que, aunque no se puede incluir a Shulze en la clase P por pertenecer a la clase L (a la cual no pertenece), podría de todos modos suceder que no se le deba excluir de la clase $\mathrm{P}$ ya que, por ejemplo, ha cometido actos obscenos en un lugar público. En otros términos, si bien parece razonable creer que no se debe condenar a Shulze puesto que él no es un ladrón, en el esquema demostrativo, desde una perspectiva lógica, se cae en la falacia de la negación del antecedente.

\section{Negación del antecedente y del consecuente. Solución replicativa}

Parece claro que habría que abandonar como solución el sendero que conduce a la negación del antecedente, aunque intuitivamente se nos presente como la más razonable. Habría que hacerlo, en efecto, si mantenemos la premisa mayor tal cual («Todos los ladrones deben sufrir una condena»). Se puede probar, no obstante, otro esquema argumentativo en el que, aun manteniendo la negación de la premisa menor, se modifica la premisa mayor. Tal es, fundamentalmente, una de las soluciones propuestas por Maarten Henket; él sostiene, en efecto, que el problema surge porque existe la tendencia general a interpretar la disposición jurídica exclusivamente como implicación material (es decir, como un enunciado que expresa una condición suficiente: $\mathrm{P} Y \mathrm{Q}$ ), mientras que -según él- es posible interpretarla también como si fuese una replicación material (es decir, enunciados que

\footnotetext{
${ }^{9}$ Naturalmente, me doy cuenta de que esta solución es la que origina una de las aporías de las que surge este trabajo. Precisamente por ello creo que la misma debe analizarse detalladamente.

${ }^{10} \mathrm{O}$ bien, formalizándolo en manera muy simple: 1) $\left.\mathrm{P} \Rightarrow(Q, 2) \neg P \therefore 3\right) \neg Q$
} 
expresan una condición necesaria: $\mathrm{P} \Leftarrow \mathrm{Q}$ ) o bien una equivalencia material (es decir, enunciados que expresan una condición necesaria y suficiente: $P \Leftrightarrow Q)^{11}$.

El esquema que resultaría de la replicación material sería: (B') «Todo aquel que debe sufrir una condena es un ladrón», «Shulze no es un ladrón», entonces «Shulze no debe sufrir una condena». En este caso -parece claro- ponemos a salvo la deducibilidad de la conclusión, pero sólo con la condición de que (y es igualmente claro) se admita el cambio en la premisa mayor, donde se afirma que todos los elementos de la clase P están incluidos en la clase L. Henket sostiene -como ya he dicho- que la disposición jurídica puede ser reinterpretada no solamente como implicación material, sino también como replicación material y como equivalencia materia $1^{12}$. Analicemos la replicación. Sabemos, en efecto, que la estructura lógica de la implicación (si... entonces) puede tener diversos sentidos, en función del tipo de condición que se encuentra en el antecedente. En el caso de la replicación (replication) el antecedente es una condición necesaria para que tenga lugar el consecuente; pero, ¿qué significa esto? En palabras simples, podemos decir que el tipo de relación establecido mediante la replicación se explica con el siguiente ejemplo. Para comprar un automóvil se necesitan (simplificando) dos cosas: dinero y voluntad. Pienso que podemos convenir en que el dinero constituye una condición necesaria de la adquisición, porque, en efecto, sin él la compra no puede ser. Por otro lado, sin embargo, es indudable que la verificación de esta condición no es en sí suficiente para que la compra se verifique, dado que se necesita también la voluntad, el deseo de comprar. Pudiera suceder, en efecto, que uno tenga el dinero pero lo utilice para realizar un viaje, por ejemplo. Si de este ejemplo trivial pasamos al examen del caso que nos interesa, podríamos preguntarnos cuál sería la formulación

\footnotetext{
${ }^{11}$ Cfr. Henket, On the Logical Analysis of Judicial Decisions, cit., pp. 155-159. Resulta evidente que en el caso del ejemplo la solución es la de la replicación; la equivalencia será analizada más adelante.

${ }^{12}$ Henket sostiene (On the Logical Analysis of Judicial Decisions, cit., p. 154) que en la literatura predomina la tendencia a analizar las normas jurídicas como implicaciones materiales. Como ejemplos de ello él cita: P. W. Brouwer, Samenhang in recht, Gröningen, Wolters Noordhoff, 1990, p. 221; M. A. Loth. Recht en taal, een kleine methodologie, Arnhem, Gouda Quint, 1984, p, 157; O. Weinberger, Rechtslogik, München, Duncker und Humblot, 1989, reimp. pp. 224. 251-53; R. Zippelius, Einführung in die juristische Methodenlehre, München. C. H. Beck, 1971, pp. 121 ss. Posiciones menos acentuadas son las de A. Soeteman, Normen Logica, Zwolle, Tjeenk Willing, 1981, pp. 355-56; I. Tammelo, Modern Logic in the Service of Law, Wien-New York, Springer, 1978, p. 77. Entre los autores que en modo explícito mencionan posibilidades diversas, junto a la implicación material de interpretar la norma, se pueden ver: U. Klüg, Juristische Logik, Berlin, Springer, 1966, reimp. pp. 128-29; H, J. Koch-H. Rüssmann, Juristische Begründungslehre, München, C. H. Beck, 1982, pp. 78 ss.: J. Rödig, «Logische Untersuchungen zur Makrostruktur rechtlicher Kodifikate», en Id. (ed.) Studien zu einer Theorie der Gesetzgebung, Berlin-Heidelberg, Springer. 1976, pp. 596-611.
} 
de una interpretación de la norma en sentido de condición necesaria, a fin de salvar la deducibilidad de la conclusión «Shulze no debe sufrir una condena». ¿Quiere decir que -como en el ejemplo de la compra- no es suficiente para la condena el que se haya cometido un delito? Como se ve, la cuestión está bastante lejos de ser nítida; en efecto, Henket, quien en un primer momento tomaba la replicación como posible solución, agrega casi de inmediato que él considera más plausible la equivalencia ${ }^{13}$.

\section{Modificación de la premisa mayor. La equivalencia}

Una ulterior posibilidad -recorrida también por Henket- es la de considerar plausible la interpretación de la disposición normativa también como equivalencia material, es decir, en otros términos, una doble implicación material ${ }^{14}$, que podemos simbolizar así: $\mathrm{P} \Leftrightarrow \mathrm{Q}$. Interpretando la disposición como equivalencia -dice Henket- se llega a una forma argumentativa lógicamente válida aun negando el antecedente ${ }^{15}$. Es cierto, en efecto, que de esta manera aseguramos la validez de la conclusión «Shulze no debe ser condenado», visto que él no es un ladrón; pero, ¿qué sucede con la premisa mayor? La disposición -afirma Henket- puede ser interpretada también como replicación y equivalencia a fin de salvar la forma lógica de una argumentación basada en la negación del antecedente. De la replicación ya he hablado antes, por lo que ahora me limito a examinar la equivalencia. Como sabemos, los estudiosos de lógica sostienen que donde existe una equivalencia material entre dos proposiciones tenemos en realidad dos proposiciones que se implican recíprocamente en modo materia ${ }^{16}$. Interpretando la disposición normativa como equivalencia material nosotros tendríamos entonces una premisa formada por dos proposiciones, en las que, alternativamente, cada una es o bien condición suficiente de la otra, o bien constituye la consecuencia. Tendríamos entonces dos premisas del tipo: a) «Todos los ladrones deben sufrir una condena» $(\mathrm{P} \Rightarrow \mathrm{Q}), \mathrm{y}$ ) «Todos los que sufren una condena deben ser ladrones» $(\mathrm{Q} \Rightarrow \mathrm{P})^{17}$.

Todo esto, si no manifiestamente absurdo, es cuando menos extraño a primera vista. Parece, en efecto, en contradicción -en líneas generales- con las formulaciones lingüísticas de las disposiciones normativas, habitualmente concebidas como el nexo de imputación de la sanción al hecho ilícito, al elemento fáctico, y no de éste a la sanción. La interpretación de la disposición

${ }^{13}$ Cfr. Henket, On the Logical Analysis of Judicial Decisions, cit., p. 156, nota 8.

${ }^{14}$ Cfr. Henket. On the Logical Analysis of Judicial Decisions, cit., p. 155-59.

${ }^{15}$ Cfr. Henket, On the Logical Analysis of Judicial Decisions, cit., p. 158.

${ }^{16}$ Ello resulta claro en todos los manuales de lógica, por ejemplo en: I. M. Copi, Introduzione alla logica, trad. it. Bologna, Il Mulino, 1969, $2^{\text {a }}$ ed., p. 307.

${ }^{17}$ En otros términos, la equivalencia $(P \Leftrightarrow Q)$ puede descomponerse en $P \Rightarrow Q$ y $\mathrm{P} \Rightarrow \mathrm{P}$. 
como equivalencia, si es cierto que garantiza la forma lógica de la argumentación a contrario, por otro lado parece generar ulteriores problemas. Si, por un lado, es razonable pensar que es suficiente que un ladrón sea tal para que deba sufrir una condena, por el otro no parece igualmente razonable afirmar que es suficiente el hecho de que alguien sufra una condena para que sea un ladrón.

Por lo menos, con la interpretación de la disposición de esta manera, emerge en modo evidente la operación valorativa (y no puramente cognoscitiva) del juez cuando él se encuentra en la condición de dilucidar el significado de la ley de cara a su aplicación. No por casualidad, en efecto, Henket se ve obligado a afirmar que «a judge who is justifying his decision is not deducing it from legal rules and so-called facts. He is tracing back the decision, regardless how he found it, to grounds that can support it» ${ }^{18}$ [ «un juez que justifica su decisión no la deduce de las normas jurídicas y de los llamados hechos. Él remonta la decisión, independientemente de cómo llegara a ella, a fundamentos que puedan apoyarla»]. La tarea del análisis lógico -concuerda Henket- «is usually seen as checking, firstly, whether the premises are consistent among themselves and, secondly, whether the conclusion can be validly deduced from the premises ${ }^{19}$ [《es normalmente vista como comprobar, en primer lugar, si las premisas son consistentes entre sí y, en segundo lugar, si la conclusión puede deducirse válidamente de las premisas»]. Sólo que, agrega, éste es un modo poco feliz (unfortunate) de presentar la cuestión, dado que sugiere, por un lado, que la lógica y la creatividad judicial nunca viajan juntas y, por el otro, que la premisa fáctica se establece prescindiendo del significado de la premisa normativa y viceversa ${ }^{20}$.

Pues bien: podemos estar de acuerdo con Henket en cuanto a los riesgos ligados al hecho de subestimar la creatividad judicial y de creer en una neta separación entre premisa normativa y premisa fáctica, rasgos que están implícitos en el análisis lógico de las sentencias. Sólo que, entonces, parecería existir una contradicción entre el intento de negar los aspectos estructuralmente ligados a la lógica (que, como sabemos, no se interesa en absoluto por la verdad material de las premisas), y la búsqueda de una solución al problema de la negación del antecedente en términos lógicos. En efecto, proponiendo una solución en términos de replicación y/o de equivalencia, Henket parece no desear salirse del ámbito lógico, si bien para justificar las fórmulas interpretativas que le sirven como base él se ve obligado a hablar ya no de lógica sino de «juridical acceptability» ${ }^{21}$ [«aceptabilidad jurídica»].

\footnotetext{
${ }^{18}$ Cfr. Henket, On the Logical Analysis of Judicial Decisions, cit., p. 162.

${ }^{19}$ Cfr. Henket, On the Logical Analysis of Judicial Decisions, cit., p. 162.

${ }^{20}$ Cfr. Henket, On the Logical Analysis of Judicial Decisions, cit., p. 162.

${ }^{21}$ Cfr. Henket, On the Logical Analysis of Judicial Decisions, cit., p. 162-163.
} 
Después de haber desarrollado su argumentación, él sostiene -no obstante- que en cuanto «logic was not invented to deduce conclusions from given premises but to find premises for given conclusions» [«la lógica no se inventó para deducir conclusiones de premisas dadas, sino para encontrar premisas para conclusiones dadas»)], ella nos pone en condiciones de individualizar las premisas a las que la conclusión puede o no referirse ${ }^{22}$.

Me parece que Henket no aclara en qué perspectiva se coloca, si en la del juez que debe decidir el caso o en la del jurista que estudia la motivación del juez. Sin embargo, si no le he malinterpretado, ya sea una u otra perspectiva, parecen ser analizadas desde un punto de vista en el que el papel de la lógica es solamente el de convalidar ex-post una decisión. Ello haría, de algún modo, que fuese claramente vana la búsqueda de solución al problema de la negación del antecedente.

\section{Negación de la premisa mayor}

A críticas más o menos semejantes también se llega si, por el contrario, en lugar de recorrer el camino de la doble implicación y de la replicación, se opta por hacer de la premisa mayor una proposición negativa, a fin de asegurar la coherencia de la conclusión «Shulze no debe sufrir la condena».

Resultaría un esquema de este tipo: (B") «Nadie que no sea un ladrón debe sufrir una condena» (o bien, en otras palabras: «Todos los que no sean ladrones no deben sufrir una condena»), «Shulze no es un ladrón», luego «Shulze no debe sufrir una condena» ${ }^{23}$. Lo que debe probarse no es que Shulze es un hombre honesto (no es un ladrón) sino la afirmación contraria, que él es un ladrón. También en este caso, como en los anteriores, emerge claramente la operación interpretativa, y no puramente declarativa, del juez, quien de una formulación normativa expresada en términos de imputación de la sanción como consecuencia del hecho de que se ha verificado un determinado hecho empírico, extrae una premisa de signo negativo donde parece imputarse una no-sanción a un no-hecho. Dicho con otras palabras: habitualmente, acerca de las formulaciones normativas existe implícitamente la idea de que cuando se verifica una determinada condición sigue una determinada consecuencia jurídica-, trasladando esta idea al ámbito del proceso judicial se podría quizá decir que tal formulación responde al ideal garantista que se resume en el concepto de presunción de inocencia; en el caso analizado lo que se debe probar en el proceso es que Shulze es un ladrón (hecho que constituye la condición para que se dé la sanción). Mediante la operación interpretativa de reformular la disposición poniendo como condición

\footnotetext{
${ }^{22}$ Cfr. Henket, On the Logical Analysis of Judicial Decisions, cit., p. 163.

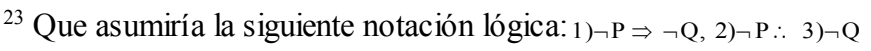


de la no-sanción el hecho de que no se haya verificado el hecho, se modifica -en mi opiniónsustancialmente la cuestión. Parecería, en efecto, que en el proceso se deba probar no (como sucedía antes) el hecho de que Shulze es un ladrón, sino que no lo es.

$\mathrm{Si}$ (y cómo) en el plano práctico todo esto tiene alguna incidencia no creo que se pueda establecer en este lugar. De cualquier modo, queda claro el hecho de que en la base de la reformulación de la disposición encontramos una operación interpretativa sustancialmente valorativa y no puramente declarativa, que, una vez más, no tiene nada que ver con la lógica.

\section{Constitutividad de la disposición jurídica}

Una estrategia que se podría seguir, en parcial alternativa a las anteriores, es la de investigar si en el ámbito de la reflexión sobre las reglas constitutivas o hipotéticoconstitutivas $^{24}$ existe algún elemento para -aun manteniendo la idea de una interpretación de la disposición jurídica sólo como implicación material- hacer de alguna manera aceptable lógicamente (y no sólo razonablemente) la conclusión de que Shulze no debe sufrir una condena $^{25}$. Tal estrategia es sólo parcialmente alternativa en cuanto que contempla de todos modos la posibilidad de una interpretación de la disposición jurídica como implicación material, es decir, como expresión hipotética donde el ilícito es solamente condición suficiente para que se dé la sanción. Las razones por las que, a fin de cuentas, esta forma de interpretación parece (al menos a mí me lo parece) más adecuada son las siguientes. En primer lugar, como ya dije antes, es evidente que si interpretamos la disposición como replicación (y entonces consideramos alilícito como condición necesaria) nos encontraríamos con una situación donde el hecho de que se verifique solamente el ilícito no da origen a la sanción; en efecto, aunque el ilícito es condición necesaria, no es -sin embargo- condición suficiente. Ello significa que para que se dé la sanción se necesita otra condición, ¿pero cuál? En segundo lugar, si se interpreta la disposición como equivalencia (y entonces consideramos al ilícito como condición necesaria y suficiente) deberíamos concluir que, por ejemplo, una prescripción jurídica que dijera más o menos «Todos los ladrones deben ser condenados» tendría el sentido de que todos quienes sufren una condena son ladrones. Una interpretación

\footnotetext{
${ }^{24}$ Recuerdo que por reglas hipotético-constitutivas se entiende aquellas reglas que ponen una condición de aquello que ellas mismas constituyen. Para profundizar en este concepto véase al menos: G. M. Azzoni, Il concetto di condione nella tipologia delle regole, Padova, CEDAM. 1988; A. G. Conte, "Materiali per una tipologia delle regole», en Materiali per una storia della cultura giuridica, 1985, 15, pp. 345-68: Id., "Costitutivita di regole», en Digesto, IV Edizione, Torino, UTET, vol. IV, 1989, 462-65.

${ }^{25}$ Esta hipótesis nació durante una discusión del problema con Paolo di Lucia.
} 
de este tipo no puede dejar de suscitar algunas perplejidades, dado que -como se sabe- quienes reciben una pena por parte de la justicia no son sólo los ladrones (sino también los asesinos, estafadores, etc.).

Es interesante notar, antes que nada, que del conjunto de las reglas constitutivas las que parecen adaptarse mejor a las finalidades de esta propuesta son las reglas hipotéticoconstitutivas, y de éstas en particular aquellas que ponen una condición de lo que ellas mismas constituyen una regla. Uso -en modo convencional- para tales reglas el sintagma «reglas hipotético-constitutivas ${ }^{26}$, aunque en realidad corresponda también a las reglas constitutivas que no ponen sino que son condición de aquello de lo cual ellas constituyen una regla ${ }^{27}$. Según el tipo de condición que ponen, los siguientes tres sub-tipos de regla hipotético-constitutiva corresponden -en mi opinión- a las tres posibilidades de las que -según Henket- dispone el juez para interpretar la disposición normativa; veamos de qué manera:

1) a las reglas anankástico-constitutivas, que ponen condiciones necesarias, correspondería la interpretación de la disposición normativa como replicación material, que se puede simbolizar: $\mathrm{P} \Leftarrow \mathrm{Q}$;

2) a las reglas metatético-constitutivas, que ponen condiciones suficientes, correspondería la interpretación de la disposición normativa como implicación material, que se puede simbolizar: $\mathrm{P} \Rightarrow \mathrm{Q}$;

3) a las reglas nómico-constitutivas, que ponen condiciones necesarias y suficientes, correspondería la interpretación de la disposición normativa como equivalencia material, que se puede simbolizar: $\mathrm{P} \Leftrightarrow \mathrm{Q}$.

${ }^{26}$ Sigo en esto a G. M. Azzoni, Il concetto di condizione nella tipologia delle regole, cit., p. 81.

${ }^{27}$ El tercer tipo de reglas constitutivas (además de las que son condición y de las que ponen condiciones de aquello de lo cual constituyen una regla) son las que presuponen las condiciones, llamadas reglas hipotéticas. Cfr. G. M. Azzoni, Il concetto di condizione nella tipologia delle regole, cit., p. 118. Naturalmente no puedo aquí referir la ya abundante bibliografía sobre las reglas constitutivas. Además de los autores ya citados se pueden ver (en la literatura italiana y sin ninguna pretensión de exhaustividad): A. G. Conte, Paradigmi d'analisi della regola in Wingenstein, en: R. Egidi (ed.), Wittgenstein. Momenti di una critica del sapere, Napoli, Guida, 1983, pp. 76-8 1; P. Pollastro. «Fenomenologia delle regole costitutive». en Materiali per una storia della cultura giuridica, 1983. 13, pp. 257-62; G. Carcaterra, Le norme costitutive, Milano, Giuffrè, 1974 (ed. provisional); Id., La forza costitutiva delle norme. Roma, Bulzoni, 1979; A. G. Conte - T. Mazzarese, «Reggole fondate su regole», en Nuovaa civiltà delle macchine, 3, 1985, 1-2 (9-10), pp. 61-63; G. di Bernardo, «Regole costitutive e prescrittive nella costruzione del sociale», en Nuova civiltà delle macchinee, 3, 1985, 3-4 (11-12), pp. 34-39: R. Guastini, «Teorie delle regole costitutive». en Rivista Internazionale di Filosofia del Diritto. 1983, 60, pp. 548-64; Id., «Norme che sono condizioni sufficienti del loro oggetto», en Materiali per una storia della cultura giuridica. 1986, 16. pp. 213-22: M. Jori, «In margine all'ultimo Conte», en Materiali per una storia della cultura giuridica, 1986, 16, pp. 443-69; T. Mazzarese, «Un libro sulle regole», en Materiali per una storia della cultura giuridica, 1984, 14, pp. 505-10: Id., «Metaregole», en Nuova civiltà delle macchine, 3, 1985, 3-4 (11-12), pp. 65-73. 
Podríamos preguntarnos si, por hipótesis, las reglas hipotético-constitutivas en general ponen constitutivamente condiciones no sólo en relación con lo que explícitamente ellas expresan, sino también, en modo implícito, condiciones de signo diferente a las expresadas en modo explícito. Intentaré explicar esto de modo más claro con algunos ejemplos.

Primer ejemplo. Supongamos que nos encontramos, en un procedimiento judicial, con el art. 45, apartado 3, del Cód. civil italiano («El incapacitado tiene el domicilio del tutor»); limitando la interpretación a la implicación material sucede que, de acuerdo con lo que se ha dicho antes, tenemos una norma jurídica que se podría ver como una regla metatético-constitutiva, que expresa una condición suficiente. Mi hipótesis tendría que ver, entonces, con la posibilidad de que, en base a lo anterior, se pueda considerar la norma, por un lado, constitutiva en positivo de la condición suficiente mediante la cual del hecho de ser un incapacitado se sigue el hecho de tener como domicilio el domicilio del tutor, y, por el otro, en negativo, de la condición suficiente mediante la cual por el hecho de no ser un incapacitado no se sigue el hecho de tener como domicilio el domicilio del tutor.

Segundo ejemplo. «El menor tiene el domicilio que corresponde al lugar de residencia de la familia o del tutor» (art. 45, apartado 2, Cód. civil italiano), o bien «La mujer que no está legalmente separada tiene como domicilio el domicilio del marido» (art. 45, apartado 2, Cód. civil italiano ${ }^{28}$. En el primer caso es bastante evidente el hecho de que racionalmente debemos aceptar que si «XX» no es un menor de edad, este hecho constituye una razón suficiente para que no tenga su domicilio en el lugar de residencia de la familia o del tutor. En modo análogo, del hecho de que «y» esté legalmente separada de «z» se sigue que «y» no tiene como domicilio el domicilio de «Z»).

Estoy hipotetizando -creo que queda claro- que la constitutividad de las reglas pueda determinarse en relación con dos dimensiones diferentes: una explícita, por lo que ellas expresan, y una implícita, por lo que ellas no expresan. En los ejemplos, por tanto, si consideramos las normas a la luz de la teoría de las reglas constitutivas tendríamos que explícitamente las normas en cuestión expresan las condiciones mediante las cuales el incapacitado, el menor y la mujer no legalmente separada tienen un cierto domicilio, pero, también, que las mismas normas expresan implícitamente las condiciones mediante las cuales los que no son menores ni incapacitados, y las mujeres separadas, tienen otro domicilio.

${ }^{28}$ El primer ejemplo lo tomo de G. Carcaterra, La forza costitutiva delle norme, cit., p. 65, y el segundo de Le norme costitutive, cit., p. 50, también de Carcaterra. El segundo lo usa también N. Bobbio, en «Norma», en Enciclopedia Einaudi, Torino, Einaudi, vol. IX, 1980, pp. 876-907 a p. 896. Véase también a G. M. Azzoni, Il concetto di condizione nella tipologia delle regole, cit., pp. 133-36. 
Me doy cuenta, naturalmente, de que la segunda dimensión (la implícita) es muy elusiva, y bastante difícil de expresar. El hecho es que -todos lo sabemos- mientras que es claro aquello que se dice (dentro de ciertos límites, por supuesto), no sucede lo mismo con lo que no se dice. Es evidente, por otro lado, que recorriendo este camino al final debemos enfrentarnos con las nociones de plenitud y de cierre del ordenamiento, nociones que expresan concepciones ni universalmente reconocidas ni unívocas, y que, sobre todo, se insertan en una determinada idea de derecho y de ordenamiento jurídico. Por desgracia (y por todas las implicaciones que tienen) no me es posible en este lugar entrar en el núcleo de la discusión de estas nociones, sobre las cuales -por lo demás- existe una abundante literatura ${ }^{29}$.

La propuesta de considerar las disposiciones normativas desde una perspectiva ligada a la teoría de las reglas constitutivas entra, evidentemente, en una concepción del lenguaje y del acto lingüístico que considera relevante el aspecto pragmático de la comunicación humana ${ }^{30}$.

Si el análisis que he efectuado, y la idea de fondo que le subyace, tienen sentido, ello podría conducir -en mi opinión- a ventajas en dos planos distintos. En el primero, se contribuiría a confirmar el potencial teorético contenido en la teoría de las reglas constitutivas, para dar cuenta de modo más completo del sentido de los actos lingüísticos mediante los cuales se expresan las disposiciones del legislador; en el segundo, manteniéndonos en el ámbito de la interpretación de la disposición normativa como implicación

\footnotetext{
${ }^{29}$ Como ejemplos cito los estudios de A. G. Conte. Además de los que ya he mencionado, se pueden ver: Saggio sulla completezza degli ordinamenti giuridici, Torino, Giappichelli, 1962; Id., «Rassegna di nuove ricerche sopra lacune e antinomie 1964-1966», en Annuario bibliografico di filosofia del diritto, 1966, 2. pp. 343-90; Id., «Completezza», en Digesto, IV Edizione, Torino, UTET; Id., «In margine all'ultimo Kelsen», en Studia ghisleriana, 1967, serie I, 4, pp. 113-25. Y también: G. Gavazzi, Delle antinomie, Torino, Giappichelli, 1959; E. Bulygin-C. E. Alchourrón, «Incompletezza, contraddittorietà, e indetorminatezza degli ordinamenti normativi», en G. di Bernardo (ed.) Logica deontica e semantica, Bologna, Il Mulino, 1977; R. Guastini, «Completezza e analogia. Studi sulla teoria generale del diritto italiano del primo Novecento» en Materiali per una storia della cultura giuridica, 1976, VI, pp. 511-91.

${ }^{30}$ Ejemplos interesantes de la aplicación de esta idea me parecen los estudios de H. Paul Grice. Cfr. «Logic and Conversation. The William James Lectures at Harvard University-Lesson II», en Syntax and Semantics-Speech Acts, P. Cole y J. L. Morgan (eds.), New York-London, Academic Press, 1975, pp. 41-58; trad. it. de M. Sbisà, «Logica e conversazione», en M. Sbisà (ed.), Gli atti linguistici. Aspetti e problemi di filosofia del linguaggio, Milano, Feltrinelli, 1978, 4ª 1993, pp. 199-219. Grice, en efecto, habla de implicaciones (implicatures) conversacionales para dar cuenta de lo que -dentro de un discurso, de ciertas reglas y de un contexto determinado- no se dice explícitamente; si, por ejemplo (p. 212), «A» está escribiendo un informe para un candidato que participa en un concurso para profesor de filosofía en Estados Unidos, diciendo: «estimado señor: Mr. -X- tiene una óptima preparación en la lengua inglesa, y ha asistido regularmente a los cursos», habría muy buenas razones para pensar que «A» quiere decir que «X» no está preparado en filosofía. De cualquier modo, naturalmente, habría que ver si del discurso conversacional podemos pasar al discurso de las disposiciones normativas.
} 
material, se podría evitar el tener que recurrir a una extensión de la interpretación como replicación y equivalencia que, a mi juicio, introducen no pocos elementos discutibles en relación con la interpretación de las disposiciones normativas. 